\section{AB0542 PRESENTATION AND OUTCOME OF LUPUS NEPHRITIS IN THE MULTICULTURAL SOCIETY OF WESTERN AUSTRALIA - A SINGLE CENTRE EXPERIENCE}

W.D. Raymond ${ }^{1}$, K. Minas ${ }^{2}$, L. Tyack ${ }^{2}$, B. Wong ${ }^{2}$, A. Douglas ${ }^{2}$, A. Kang ${ }^{3}$, D. Wong ${ }^{3}$, A. Chakera ${ }^{1,2}$, J. Nossent ${ }^{1,4} .{ }^{1}$ School of Medicine \& Pharmacology, University of Western Australia; ${ }^{2}$ Renal Medicine; ${ }^{3}$ Pathology; ${ }^{4}$ Rheumatology, Sir Charles Gairdner Hospital, Perth, Australia

Background: Lupus Nephritis $(L N)$ is one of the most severe complications that can occur in patients with Systemic Lupus Erythematosus (SLE) as it increases the risk of renal failure, comorbidity and death. As with SLE, there is a significant impact of ethnicity on LN severity and outcome.

Objectives: To investigate the impact of ethnicity of $L N$ presentation and health outcomes in a tertiary referral centre in Western Australia.

Methods: Single centre retrospective cohort study of 104 patients with biopsy confirmed lupus nephritis (LN) collating clinical characteristics, renal histopathology, serology, medication use, disease activity (Systemic Lupus Erythematosus Disease Activity Index - 2K (SLEDAI-2K)), organ damage (SLICC-Damage Index $(\mathrm{SDI})$ ), and clinical outcomes from index biopsy event to the last visit. Outcomes included the high serum creatinine $(\geq 150 \mathrm{umol} / \mathrm{L})$, low eGFR $(<50 \%)$, the need for renal replacement therapy (RRT), and death. Outcomes were assessed across ethnicity with comparative statistics, Chi-square, and survival analysis.

Results: Asian $(n=17,16.3 \%)$, Caucasian $(n=79,76.0 \%)$ and Indigenous $(n=8$, $7.7 \%)$ patients were similar for age $(\mathrm{p}=0.149)$, gender $(\mathrm{p}=0.309)$ and SLEDAI-2K ( 8.5 vs. 9.0 vs. $13.0, p=0.897$ ), non-renal SLEDAl-2K ( 10.5 vs 2 vs. $1, p=0.528$ ). Time to biopsy from the initial SLE diagnosis was shorter in Asian and Indigenous patients $(p=0.055)$. At the index biopsy, ethnic groups were similar for WHO Class distribution (predominantly Classes 3 and $4, p=0.345$ ) and clinical signs of renal disease (the presence of haematuria, proteinuria, pyuria and/or red cell casts) $(\mathrm{p}=0.874)$. Indigenous patients trended towards higher creatinine $(66.0$ vs 71.0 vs. $101.5, p=0.214$ ), lower eGFR ( $60 \%$ vs. $60 \%$ vs. $32.5 \%, p=0.076$ ), lower $\mathrm{C} 3$ ( 0.67 vs. 0.65 vs. $0.47 \mathrm{~g} / \mathrm{L}, \mathrm{p}=0.375$ ) and $\mathrm{C} 4$ ( 0.15 vs 0.12 vs $0.03 \mathrm{~g} / \mathrm{L}, \mathrm{p}=0.272$ ), and higher anti-dsDNA antibody levels ( 16.0 vs 26.5 vs $520.0, p=0.81)$, albeit non-significantly (due to low numbers).

At the last follow-up, 6.4 years (IQR 3.8, 12.4) after the index biopsy, $10 \%$, $30 \%$ and $12.5 \%$ of Asian, Caucasian and Indigenous patients had high serum creatinine levels ( $\geq 150$ umol/L) $(p=0.434)$ and $10 \%, 28.9 \%$ and $25.0 \%$ had low eGFR $(<50 \%)$ respectively. Chronic renal replacement therapy (RRT) was needed in $0 \%, 5.1 \%$, and $12.5 \%$, respectively $(\mathrm{p}=0.386)$, while death occurred in $0 \%$, $8.7 \%$ and $25.0 \%$, respectively $(p=0.115)$. Indigenous patients had the poorest survival rates at 1,5 and 10 years compared to Caucasian patients $(\mathrm{p}=0.002)$. Conclusions: This interim analysis demonstrated that Asian patients had a better prognosis regarding serum creatinine, eGFR, commencement of RRT and mortality, compared to Caucasian and Indigenous patients. Indigenous patients were both over-represented in this cohort and showed poorer prognosis in terms of RRT and mortality rates compared to Asian or Caucasian patients in WA.

Disclosure of Interest: None declared

DOI: 10.1136/annrheumdis-2017-eular.5854

\section{AB0543 ANXIETY AND DEPRESSION IN CHINESE PATIENTS WITH PRIMARY SJÖGREN'S SYNDROME}

Y. Cui ${ }^{1}$, L. Li ${ }^{1}$, Q. Zhao ${ }^{1}$, S. Chen ${ }^{1}$, L. Li ${ }^{2}$, Z. Gu ${ }^{1} .{ }^{1}$ Department of Rheumatology, Affiliated Hospital of Nantong University; ${ }^{2}$ School of Nursing, nantong University, Nantong, China

Background: Primary Sjögren's syndrome (pSS) is a multisystem autoimmune disorder characterised by lymphocytic infiltration and exocrine failure of salivary and lacrimal glands, resulting in the classical symptoms of the disease including xerostomia (dry mouth) and keratoconjuctivitis sicca (dry eyes). pSS has the potential to impair both psychological state and the health-related quality of life (HR-QOL). A common mental health problem among adults with pSS is anxiety and depression. In addition, depression is more common in pSS than in the general population and has been associated with enhanced fatigue, reduced health-related quality of life, increased levels of physical disability and increased health care costs. Besides, depressed pSS patients have poorer long-term outcomes, including more complications. Anxiety was more common than depression in pSS. The most affected domains were vitality in the SF-36 and general/physical fatigue in the MFI. Extraglandular systemic involvement was not a major determinant of QoL alteration in patients with pSS.

Objectives: Prevalence of anxiety and depression are high in women with Primary Sjögren's syndrome (pSS). Our aim was to compare anxiety and depression in pSS patients and healthy controls and evaluate its relationship with the disease activity, sleep and quality of life; as well as to analyze potential determinants of anxiety and depression.

Methods: Sixty-seven patients fulfilling the American-European Consensus Group criteria for pSS (mean age 52.76 years (s.d. 13.16)) and 42 age-matched healthy controls were included. Participants completed self-administered questionnaires, namely Hospital Anxiety and Depression Scale (HADS), Short Form 36 (SF36) scores and the Pittsburgh Sleep Quality Index (PSQI). In addition, the European League Against Rheumatism Sjögren's Syndrome Disease Activity Index (ESSDAl); Patient Reported Index (ESSPRI) and systemic inflammation (erythrocyte sedimentation rate (ESR), C-reactive protein (CRP) level) were recorded in patients. Independent samples t-tests, $\times 2$ analyses and logistic regression modeling were used to analyze the data.

Results: pSS patients experienced greater anxiety and depression than controls (HADS-A scores: mean \pm SD $6.5 \pm 3.4$ for pSS versus $3.8+3.4$ for controls; $P$ $=0.002$. HADS-D scores: mean \pm SD $6.7 \pm 4.1$ for pSS versus $4.2 \pm 3.5$ for controls; $P=0.012$ ). And there were significant correlations among fatigue, pain, disease activity, dryness, sleep, quality of life and anxiety/depression. Meanwhile, logistic regression analysis revealed that poor quality of life and ESSPRI were significantly associated with anxiety/depression in pSS patients.

Conclusions: The study suggests that optimal care of pSS patients may include the detection and management of anxiety and depression. Early recognition and appropriate intervention is therefore essential to reduce the negative impact of anxiety and depression on the patient's quality of life and outcome of their disease. Acknowledgements: This research was supported by grants from the National Natural Science Foundation of China $(81471603,81172841)$; “Top Six Types of Talents" Financial Assistance of Jiangsu Province (Grant no. 10.WSN016). Disclosure of Interest: None declared

DOI: 10.1136/annrheumdis-2017-eular.3069

\section{AB0544 LEVELS OF VITAMIN D IN A COHORT OF PATIENTS WITH SYSTEMIC LUPUS ERITHEMATOSUS. RELATIONSHIP WITH DISEASE ACTIVITY AND BONE MASS}

Y. Garcia-Mira, S. Heredia, M. Martínez-Morillo, L. Gifre, A. Prior-Español, J. Camins, J. Cañellas, S. Holgado, L. Mateo, A. Olivé. Hospital Universitari Germans Trias i Pujol, Badalona, Spain

Background: Vitamin $D$ (vit D) is an immunoregulatory hormone which seems to mediate immune tolerance. Several studies have suggested that vit $D$ deficiency may be related to increased disease activity in patients with systemic lupus erythematosus (SLE).

Objectives: To analyze 25-hydroxyvitamin D (25-OHD) levels in a cohort of patients with SLE and to investigate their relationship with clinical, analytical, immunological and densitometric parameters.

Methods: Prospective study including patients with SLE (according to SLICC criteria 2013) performed in an Academic Hospital with a referral area of 800.000 inhabitants. 152 patients with SLE were included (138 women [46 postmenopausal]/14 men) with a mean age of $46 \pm 12$ years (range: $20-75$ ). Clinical parameters (including risk factors for osteoporosis, presence of skeletal fractures, treatment with glucocorticoids (GCC) as well as SLE involvement including haematologic, renal, neurological and skin), biochemical determinations (including 25-OHD, parathormone (PTH)), immunological (ANA, DNA and complement) and SLE activity and severity (assessed by SLEDAI and SLICC index) were assessed in all patients. Bone mineral density was performed by DXA (at lumbar spine and proximal femur). Vit $D$ deficiency was defined as $25-O H D$ values under $20 \mathrm{ng} / \mathrm{mL}$. Low bone mass was considered as T or Z scale $<-1 \mathrm{SD}$; and osteoporosis as $T$ $<-2.5 \mathrm{SD}$ [age $>50$ years] or $Z<-2 \mathrm{SD}$ [age $<50$ years]. The study was approved by the Clinical Research Ethics Committee and all patients provided informed consent to participate. Statistical analysis was performed by SPSS.20.

Results: The mean values of 25-OHD were 19.8 \pm 11.4 (range, 4.2-66.6). $87.5 \%$ of patients had $25-\mathrm{OHD}$ levels below $30 \mathrm{ng} / \mathrm{mL}, 61.2 \%$ below $20 \mathrm{ng} / \mathrm{mL}$ and $15.1 \%$ below $10 \mathrm{ng} / \mathrm{mL}$. The lowest values were in winter (80\%) and spring (64.3\%). $42.8 \%$ of patients received vitamin D supplements. $56.5 \%$ of patients had low bone mass ( $T$ or $Z$ scale $<-1$ SD), and $15.8 \%$ had osteoporosis. Levels of 25-OHD showed no correlation with SLE disease activity (complement, Ac antiDNAds, SLICC/SLEDAI) neither with bone mass by DXA. Patients with low bone mass ( $T$ or $Z<-1$ SD scale) were older (at the time of inclusion and age of SLE diagnosis), had higher SLICC and lower complement levels whereas no differences were observed in SLEDAI and $25-\mathrm{OHD}$ values. $37.5 \%$ of the patients were treated with GCC. Patients without GCC treatment had higher prevalence of vit D deficiency $(73.9 \%$ vs. $55.6 \%, p=0.034)$ compared to patients with GCC treatment.

Conclusions: $61.2 \%$ of patients with SLE have $25-\mathrm{OHD}$ deficiency, which is more frequent in winter and spring, and mostly in those patients without GCC treatment. 25-OHD values showed no correlation with the disease activity and damage. However, patients with low bone mass had higher SLICC and hypocomplementemia. Thus, our results suggest the need to perform clinical guidelines to assess bone mass and bone metabolism in this clinical condition Additionally, we recommend quantifying vit D levels in winter/spring and don't forget to assess those patients without GCC treatment.

Disclosure of Interest: None declared

DOI: 10.1136/annrheumdis-2017-eular.5349

\section{AB0545 EFFECT OF EMPATHY NURSING ON THE LIFE QUALITY OF THEPATIENTS WITH SYSTEMIC LUPUS ERYTHEMATOSUS}

Y. Wen ${ }^{1}$, L. Yan ${ }^{1}$, L. LiMin ${ }^{2} .{ }^{1}$ Department of Rheumatology; ${ }^{2}$ Nursing department, Third Affiliated Hospital of Sun Yat-sen University, Guangzhou, China

Background: The patients with Systemic Lupus Erythematosus always had subhealth status in psychology and been troubled by the chronicity of the disease. Empathy nursing is an effective treatment for chronic disease such as cancer or terminal patients. 